\title{
X-Linked Ichthyosis
}

National Cancer Institute

\section{Source}

National Cancer Institute. X-Linked Ichthyosis. NCI Thesaurus. Code C84779.

The second most common form of ichthyosis. It is an X-linked inherited disorder with mild skin manifestations. The skin changes appear at birth and include keratinization and scaling. 